\title{
General Dental Practices With and Without A Dental Therapist: A Survey of Appointment Activities and Patient Satisfaction With Their Care
}

E. Barnes, A. Bullock, J. Cowpe, K. Moons, W. Warren, D. Hannington, M. Allen, I. G. Chestnutt, S. Bale and C. Negrotti

\begin{abstract}
Introduction

Policy changes regarding the role of Dental Hygienist-Therapists (DTs) have been implemented in recent years with a view to promoting delivery of oral health-care through a more preventive-focussed, team work approach.
\end{abstract}

\section{Aims}

To explore i) treatments led by dentists and DTs, and ii) patients' satisfaction with the care they received.

\section{Materials and Methods}

Six case-studies of general dental practices in Wales, UK: three with and three without a DT. For each participant, a patient-satisfaction questionnaire and a staff-member-completed appointment record form were completed. We sought to recruit 150 patients seeing a dentist and 100 patients seeing a DT, per case-study practice.

\section{Results}

1224 patients were recruited in total (314 DT and 910 dentist appointments). Preventive work accounted for nearly half of all treatments. Dentists, in practices with a DT, undertook significantly less preventive and restorative work, and significantly more extractions and advanced treatment $\left(\mathrm{p}<0.005, \chi^{2}=15.352\right)$. Patient satisfaction and confidence in dentists' or DTs’ ability was uniformly high (97\% and 99\% each group respectively). 


\section{Conclusion}

Practices with DTs provided a more preventive-focussed approach to oral health-care delivery; dentists in these practices performed more complex work. Positive patient satisfaction and confidence in practitioners' ability suggest patient acceptability of a preventive model.

\section{Introduction}

Increasing population health suggests that demand on primary care for general dental practitioner examinations with little or no further treatment will increase with time. ${ }^{1}$ However, improvements in global dental health also means that an elderly population retaining dentition for longer ${ }^{2}$ is leading to a parallel population with complex treatment needs. ${ }^{3}$

The mix of skills in the dental workforce has been extensively debated for many years. ${ }^{4}$ With their extended duties, ${ }^{5}$ there is considerable scope to delegate routine examinations and restorations to dual-qualified dental hygienist-therapists or singly-qualified dental therapists (we use the abbreviation DTs to refer to either single or dual-qualified hygienist-therapists). ${ }^{6}$,

${ }^{7}$ Research detected that $73 \%$ of clinical time was spent carrying out tasks that could have been delegated to dental care professionals (DCPs). ${ }^{8}$ One study examined visits by type and found that $35 \%$ of restorative intervention patient visits were duties that could be provided by hygiene-therapists, accounting for $43 \%$ of clinical time, while delegation of diagnostics and treatment planning would account for $70 \%$ of patient visits and $58 \%$ of clinical time. ${ }^{6}$ By providing routine care to patients, DTs can release the dentist for more complex cases.

'Direct Access' arrangements ${ }^{9}$ mean that both singly-qualified dental hygienists (DHs) and DTs can diagnose, treatment plan, and undertake their full scope of practice ${ }^{5}$ without working to a dentist's prescription. Unfortunately, current funding regulations are linked to dentists 
with a performer number, denying NHS patients direct access to DHs and DTs in the general dental service (GDS). These regulations do not apply to patients treated privately in the GDS or in the community dental service. In 2015, almost 250 DHs/DTs were carrying out 'Direct Access' privately within general dental practices (GDPs). ${ }^{10}$ In addition, in both NHS and private practices DH/DTs are only able to prescribe prescription-only medicines (e.g. local anaesthetic) or apply topical fluoride either under the direction of a dentist or with a Patient Group Directive (PGD) in place. PGDs are dentist-written instructions, signed off by a pharmacist, specifying named medications and the circumstances in which they would be prescribed. ${ }^{11}$ Applying for a PGD has been noted to be a lengthy and difficult process ${ }^{12,13}$ and the specified circumstances for prescribing are restrictive in practice. ${ }^{13}$ Following appropriate training, DTs can prescribe radiographs however dentists currently have to review the film to close the process. ${ }^{13}$ Other barriers limiting the employment of DTs in the GDS include knowledge of DTs’ remit, concern about patient acceptance, accommodation, availability and supervision. ${ }^{14-16}$ Proposed new models of funding emphasising prevention provide promising opportunities for DTs. ${ }^{17,18}$ Although these have promoted acceptance of greater use of DTs in oral healthcare, skill-mix development has progressed slowly. ${ }^{14,19}$

Health service studies reveal benefits of team work. When staff 'only do what only they can do' team skill-mix is optimised. ${ }^{20}$ The present study uses a case study method to explore the range of activities carried out in GDPs, the potential scope for delegation to DTs and patients' satisfaction with their care.

\section{Materials and methods}

Six NHS GDPs across South Wales were selected as case studies. These were purposively sampled, informed by the research team and Advisory Group’s knowledge of practices 
suitable for investigation. We judged that it was appropriate to seek to recruit established GDPs with different models of skill-mix. GDPs were selected from across three Health Boards in South Wales to explore different demographic and regional populations and practices; two practices from each Health Board, one with a DT and one without. The selected practices also included a variety of GDS settings, including from the corporate bodies.

Between July and November 2016, members of the research team, supported by research nurses from Cwm Taf and Aneurin Bevan University Health Boards, attended the practices. With consent, two questionnaires were completed at each appointment - a patient satisfaction questionnaire and a staff member-completed appointment record form. The researchers attended only on days when both dentist and DT were practicing.

The record sheet recorded whether the appointment was part of a course of treatment and what actions were taken. We provided a simple classification of actions so that these record forms could be completed by the dentist/DT or attending dental nurse: check-up only (diagnosis), preventive action, and treatment (with a simple sub-classification for restorations, extractions, advanced treatment). We also requested an indication of whether the action could have been carried out by a DT or another dental team member and if so, reasons for not delegating.

The patient-satisfaction questionnaire was informed by available questionnaires ${ }^{21,22}$ and by a structured review of the available literature. It included perceptions of the quality of treatment, confidence in different dental team members, views on appointment waiting time, cost, and so forth. Drafts of both forms (see supplementary material) were discussed with the project Advisory Group and piloted with dental professionals/patients outside the case study sites to establish face validity. 
From each practice, we aimed to recruit 150 patients seeing a dentist and 100 patients seeing a DT, a total of 1200 participants (900 dentist/300 DT). Fewer participants seeing DTs were recruited to reflect the balance of work within the practices. While many practices had more than one dentist working full-time, DTs were mainly employed on a part-time basis often with one DT working only two or three days.

The completed appointment record sheets and questionnaires were collated by the researchers and entered into SPSS. Descriptive statistical analysis and Pearson's Chi Squared tests were performed to explore differences and associations within the different practice models or between professional roles. In some practices with DTs, patients saw both the dentist and the DT; separate record forms were completed by the dentist and DT however, the patient completed one satisfaction form for both appointments. The analysis of the patient satisfaction forms were analysed for three categories: those seeing the dentist, those seeing a DT and those seeing both a dentist and a DT.

\section{Results}

1224 patients were recruited from six sites; 314 saw a DT, 910 a Dentist. 114 participants attended joint appointments (dentist and DT). Table 1 provides a practice staffing summary.

Table 1: Staffing profiles of case study practices

\begin{tabular}{|c|c|c|c|c|c|c|}
\hline $\begin{array}{l}\text { Case } \\
\text { study } \\
\text { site }\end{array}$ & Dentists & $\begin{array}{l}\text { Foundatio } \\
\text { n Dentists }\end{array}$ & $\begin{array}{l}\text { Dental } \\
\text { Therapist }\end{array}$ & $\begin{array}{l}\text { Dental } \\
\text { Hygienist }\end{array}$ & $\begin{array}{l}\text { Dental } \\
\text { Nurse }\end{array}$ & $\begin{array}{l}\text { Orthodonti } \\
\text { st }\end{array}$ \\
\hline $\mathbf{1}$ & 5 & 1 & $2(\mathrm{p} / \mathrm{t})$ & $1(\mathrm{p} / \mathrm{t})$ & 8 & 0 \\
\hline $\mathbf{2}$ & 5 & 0 & $1(\mathrm{p} / \mathrm{t})$ & 1 & 6 & $1(\mathrm{p} / \mathrm{t})$ \\
\hline $\mathbf{3}$ & 2 & 0 & $2(\mathrm{p} / \mathrm{t})$ & 0 & 5 & 1 \\
\hline $\mathbf{4}$ & 4 & 0 & 0 & $1(\mathrm{p} / \mathrm{t})$ & 5 & 0 \\
\hline $\mathbf{5}$ & 3 & 1 & 0 & 0 & 6 & 0 \\
\hline
\end{tabular}




\begin{tabular}{|l|l|l|l|l|l|l|}
\hline $\mathbf{6}$ & 3 & 1 & 0 & 0 & 5 & 0 \\
\hline
\end{tabular}

Participants were mainly adults (88\% of dentist appointments and 85\% of DT appointments); practices without a DT saw statistically significantly more children (16\%) than practices with $(11 \%)\left(p<0.05, \chi^{2}=5.053\right) .87 \%$ were routine appointments, and $13 \%$ were emergency appointments; practices without a DT saw statistically significantly more emergency/urgent appointments $(16 \%)$ than practices with $(12 \%)\left(p<0.05, \chi^{2}=3.864\right)$. Regular examinations accounted for $36 \%$ of appointments, $64 \%$ were receiving treatment; dentists in practices with a DT carried out statistically significantly more examinations (54\%) than practices without a DT $(41 \%)\left(p<0.001, \chi^{2}=14.165\right)$. For those receiving treatment, $60 \%$ were undergoing a course of treatment; $36 \%$ at first appointments and 24\% at later appointments.

83\% of appointment record forms were for NHS appointments; of patient-satisfaction questionnaires $77 \%$ seeing the dentist were NHS only, $68 \%$ of those seeing the DT. Nearly half of all NHS respondents had nothing to pay, ranging from $37 \%$ in one case site to $67 \%$ in another. DTs were statistically significantly more likely to be delivering private care than dentists $\left(p<0.001, \chi^{2}=26.209\right)$. There was no significant association between payment type and treatment type carried out by DTs.

\section{Appointment records}

The type of treatment activity carried out in both practice types were explored. Practices with DTs carried out more preventive treatment (50\%) (including periodontal treatment) than those without a DT (27\%). Those without DTs carried out more restorative work overall (50\% without a DT, 32\% with a DT). Practices without a DT carried out marginally more extractions, advanced treatments and other treatments (Figure 1). 


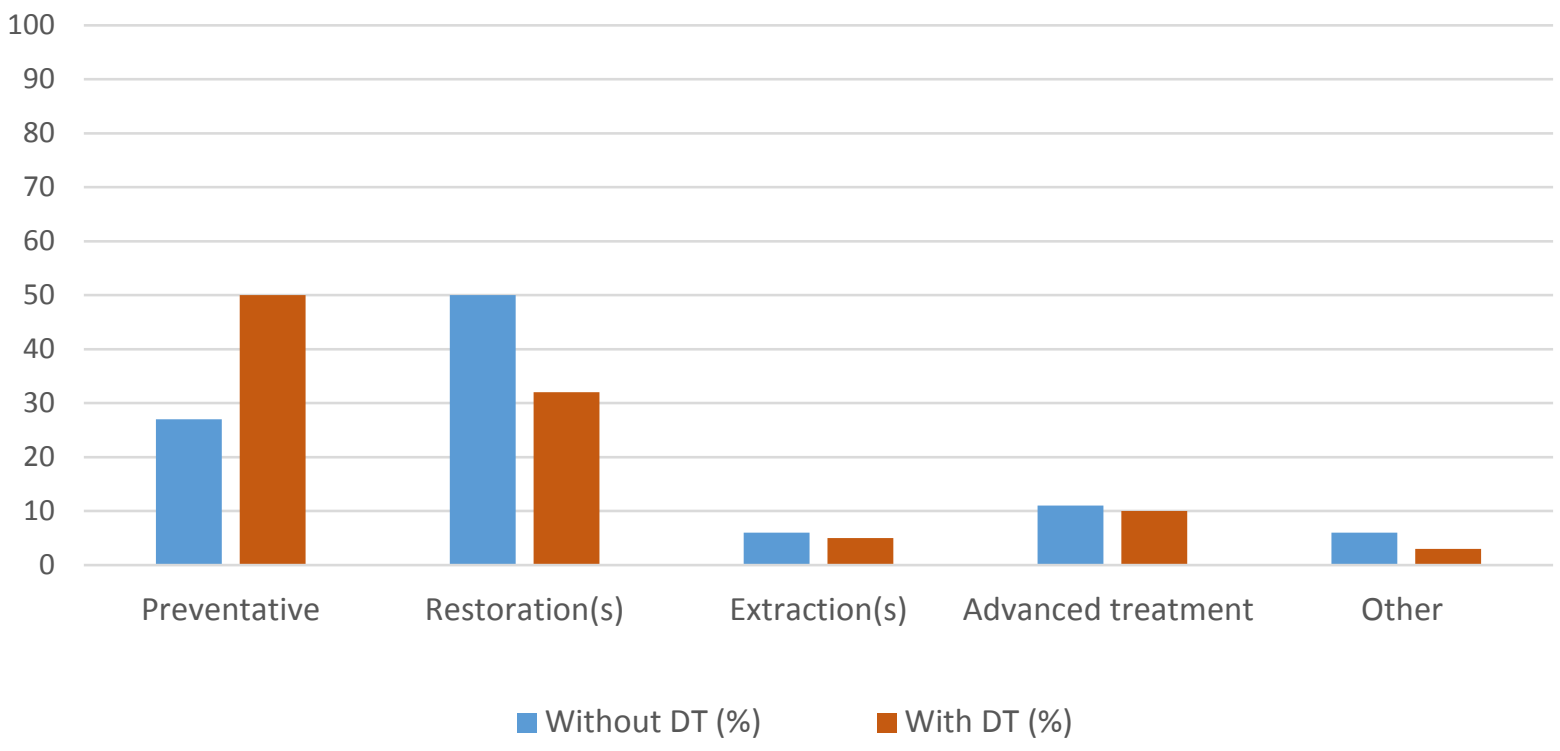

Figure 1: Activities carried out in practices with or without Dental Therapists.

When the data were explored by job role, the results showed that dentists with a DT carried out less preventive work than those without a DT (26\% without a DT, $18 \%$ with a DT) (Figure 2). Preventive treatment (including periodontal work) accounted for nearly threequarters of DTs workload (72\%). While dentists without DTs carried out more restorations (50\% without a DT, 43\% with a DT), dentists with a DT carried out more extractions (6\% without a DT, $11 \%$ with a DT), and Other treatments (6\% without a DT, 7\% with a DT) and nearly double the amount of advanced treatments than those without a DT (12\% without a DT, $21 \%$ with a DT).

Further analysis showed that in practices with a DT, dentists were doing significantly less preventive and restorative work, and significantly more extractions and advanced treatment than dentists without a DT $\left(\mathrm{p}<0.005, \chi^{2}=167.771\right)$. 


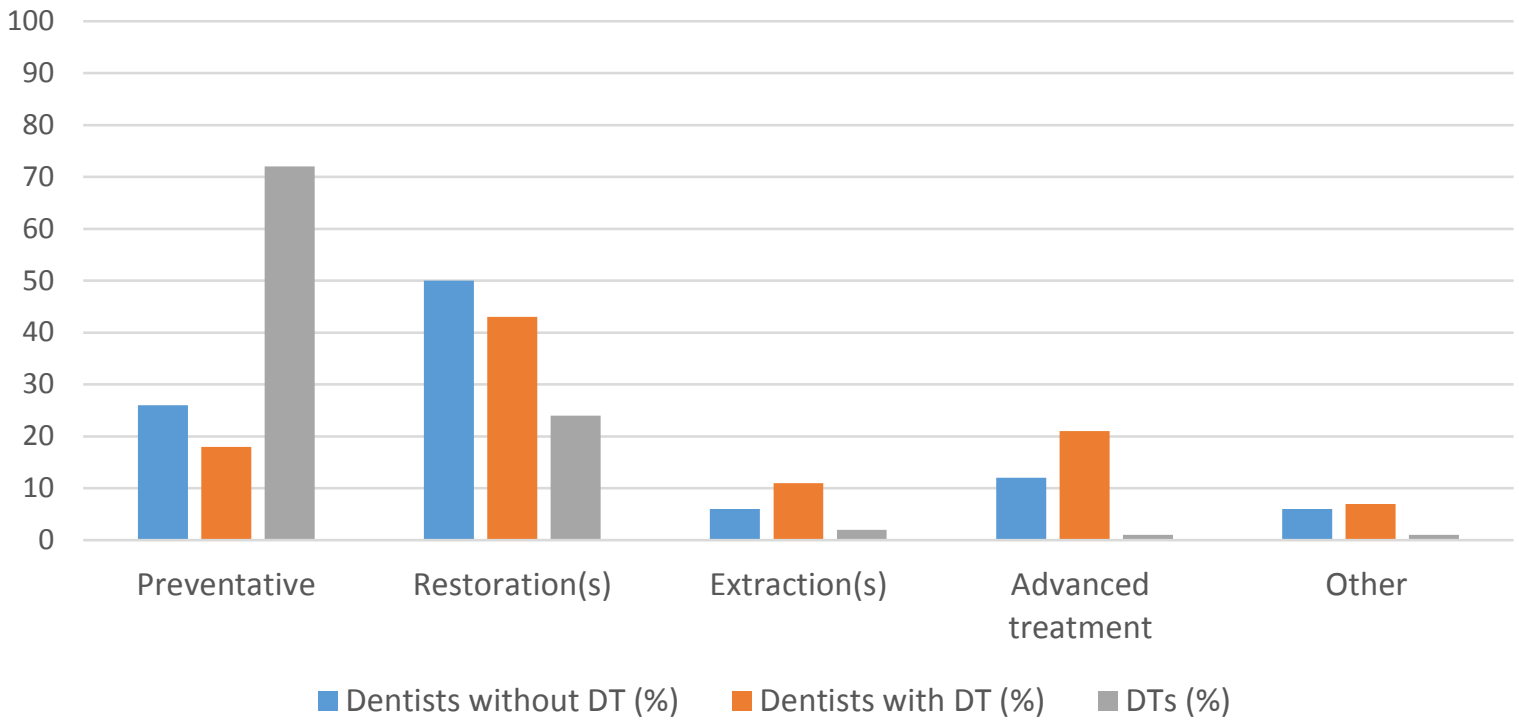

Figure 2: Activities carried out by job role in practices with or without Dental Therapists.

Participants were asked to indicate whether the appointment could have been carried out by a DT. A larger proportion of appointments with dentists in practices without a DT reported that the appointment could, in theory, have been completed by a DT (37\% without, 27\% with). More without DTs were unsure whether a task could be delegated (6\% without DTs, $2 \%$ with DTs). Appointments that dentists with DTs were unsure about were mainly check-ups (80\%) while dentists without were almost equally unsure about check-ups (48\%) and treatments (52\%). Statistical analysis showed that practices with a DT were statistically significantly more likely to be unsure about preventive tasks, while those without were unsure about restorative, extractions, or advanced treatments $\left(p<0.001, \chi^{2}=20.844\right)$.

Dentists with a DT were asked to indicate why the appointment had not been delegated. The appointment being outside the DT’s scope of practice was the most frequently identified reason (61\%); check-ups accounted for 72\% of appointments identified as outside DTs' scope of practice. Patient preference (11\%) or challenging patients (3\%) were in the minority. In $6 \%$ of cases it was because the DT was not available. "Other” responses included an error in 
booking $(n=4)$, being a first appointment and therefore unsure whether the work needed was within their scope of practice $(n=3)$, or because it was a check-up/dentist needed to open the treatment plan (10\%).

\section{Patient satisfaction}

Across all practices, $81 \%$ of patients seeing the dentist, $86 \%$ of those seeing the DT and $78 \%$ of those who saw both dentist and DT said the appointment was running to time. Patients were statistically significantly more likely to report that their appointment was with their usual dentist in practices with a DT (62\%) compared to those without a DT (38\%) $(\mathrm{p}<0.005$, $\left.\chi^{2}=8.391\right)$. Practices with a DT were also statistically significantly easier to arrange an appointment with $(65 \%)$ than those without $(35 \%)\left(\mathrm{p}<0.005, \chi^{2}=12.545\right)$. Within practices with a DT, there were no significant associations between professional role and reported ease of making appointments.

Overall, significantly more patients seeing a DT on their visit reported being given oral health advice - $91 \%$ of appointments with a DT, $86 \%$ seeing the dentist, and $98 \%$ seeing both $\left(\mathrm{p}<0.005, \chi^{2}=16.331\right) .82 \%$ of patients seeing the dentist, $78 \%$ seeing the DT and $89 \%$ seeing both felt that the appointment was unhurried. Of patients paying for treatment, 85\% seeing the dentist, $88 \%$ seeing the DT and $98 \%$ seeing both reported that the cost was acceptable. There was no statistical significance between acceptability of payment and the professionals seen.

Overall confidence in the dentists’ or DTs' ability was high, with patients reporting either 99\% (dentists, practices without DTs) or 100\% (DTs/both, practices with DTs) confidence in the professionals delivering their care. 
Although still high, those who saw a DT showed the lowest percentage of "very satisfied" responses (72\%). However, those who saw both a dentist and DT accounted for the highest rate of selection (91\%) (Figure 3). Overall satisfaction (satisfied or very satisfied) was around 97\% for each participant group.

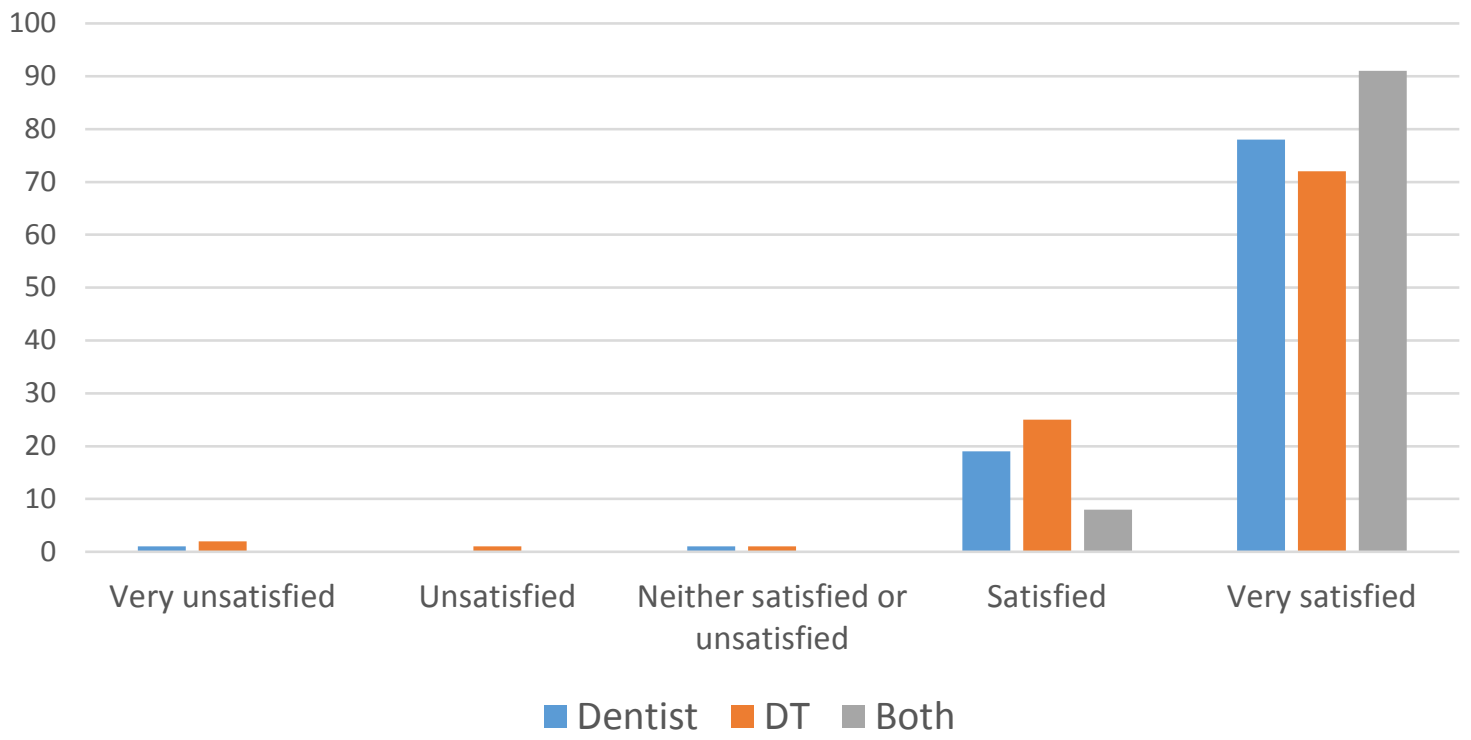

Figure 3: Patients' satisfaction with care received

There were no statistically significant differences in either confidence or satisfaction with their care between professional roles within practices, or between DT and no-DT practices.

\section{Discussion}

Practices with DTs were able to provide a more preventive-focussed approach to patient care and dentists in practices with DTs carried out a greater proportion of complex treatments. However, a greater proportion of DT's work was funded privately. Dentists without a DT were slightly more uncertain about whether an appointment could be completed by a DT; but dentists with a DT were still unsure about some preventive work. Patients in practices with DTs reported that appointments were easier to secure, and it was easier to see their preferred 
dentist. More of those seeing a DT reported receiving oral health advice than those seeing only a dentist. High reported patient satisfaction and confidence in practitioners’ ability suggests that patient acceptability of a team-based preventive model is not a barrier.

This study has limitations which should be taken into account when drawing conclusions. Firstly, the data reflects self-reported responses from a small sample of practices. We allowed dentists and DTs to use their professional judgement in categorising the type of treatment carried out as the appointment record forms were completed in between appointments and we did not want to overburden them with too much information. We acknowledge that this may cause some minor variation in responses. Although data was gathered from a small sample of practices, the practices themselves reflected different models of skill-mix, with data being collected over a period of time - sometimes over a few months. As such we believe we captured a snapshot of these practices' typical workload which accurately reflects their operation. As well as different models of skill-mix, the practices also demonstrated varying staffing sizes and UDA output. In this study we were aiming to explore diversity (operation, geography, socio-economic areas, etc.) within mainly NHS-funded practices, rather than attempt to provide matched cases for comparison. The overlap in patient-satisfaction questionnaires for those seeing the Dentist/DTs may have masked nuances in the patients' experiences of the two appointments. However, as the results were more positive than for single-professional treatment it suggests that patients' experiences of these appointments were slightly different. Within the appointment record forms, staff members were asked to indicate whether the appointment could have been carried out by a DT - in practices without a DT this may have been confusing. Participants with no experience of working alongside a DT may not have a clear knowledge of their scope of practice, therefore we have not drawn as many conclusions from their responses. The patient-satisfaction questionnaires also relied on patient self-reporting, immediately following appointments and completed within the 
practice. To minimise any bias, all discussion about the study and the data gathering process was with a non-staff member researcher and patients were reassured that the forms were completely confidential.

The finding that the presence of a DT allows the practice to provide more preventive treatments and frees up the dentist for more complex work is in line with the wider international literature on skill-mix. ${ }^{10,23}$ Patients also reported that it was easier to get an appointment, and with their preferred dentist. UK and USA-based reviews also found evidence that inclusion of DTs in the dental team improved patient access. ${ }^{24,25}$ This improvement may potentially be most felt in underserved populations (e.g. younger and older patients) ${ }^{23,26}$ and help reduce health inequalities. ${ }^{27,28}$

Encouragingly, patient preference accounted for only $11 \%$ of tasks that were not delegated in practices with a DT. This coupled with slightly higher reported patient confidence when seeing either a DT or both a dentist and DT suggests patients value a team work approach to their care. Dentist concerns over patient acceptability has been reported as a barrier to delegation, ${ }^{29}$ however, the evidence indicates that patients seeing DTs reported higher satisfaction with their care. ${ }^{24,25,30,31}$

Dentists without a DT were slightly more uncertain about whether an appointment could be completed by a DT; but dentists with a DT were still unsure about whether some tasks, mainly preventive work, were within the DTs' scope of practice. Even among practices with DTs knowledge of their full scope of practice may have been a factor. While the literature has shown that dentists are sometimes unsure of the full scope of DTs' clinical remit, ${ }^{29,} 32,3372 \%$ of these appointments were check-ups, therefore it is possible that there was some confusion between being outside the scope of practice and not being allowed under the current NHS contract. 
That dentists in practices with a DT carried out more examinations may be explained by the current NHS contract requirements or other regulations (e.g. DH/DTs' ability to prescribe prescription only medicines, requiring a dentist to review radiographs).${ }^{13}$ Despite wider changes to enable the use of team work, little consideration has been paid to the funding mechanism to support and encourage team working. ${ }^{4}$ The contract changes regulating dental remuneration, introduced in 2006, have been reported to discourage referral to DTs, ${ }^{3,34}$ and restrictive rules and regulations on DTs' activity have hindered direct access, ${ }^{13}$ maintaining use of the most expensive clinicians to treat a population with improving oral health. ${ }^{35}$ and providing an unnecessary extra burden to practitioners and patients. Ward ${ }^{16}$ states that clinical governance needs to be reconfigured and developed to address DTs' extended roles, bringing the current challenges to implementation into focus. Practices committed to team working have to find innovative ways of working to overcome these difficulties ${ }^{36}$ resulting in diverse payment systems and working practices for DTs which may have implications for recruitment and retention. ${ }^{37}$

Practices with a DT also completed a higher number of private appointments; this also has some implications. While the majority of patients were happy with the costs incurred during their treatment, access to services is an important priority for tackling health inequality. ${ }^{26}$ Those who would benefit most from a preventive team approach to patient care may not be those able to access private care. The current Welsh General Dental Service Contract Reform programme emphasises team-working to deliver, and appropriately remunerate, preventive care. ${ }^{38}$ It should also be considered that having a DT working privately influences the treatment decisions made by dentists, creating more appointments and wider opportunities for DTs to complete their scope of practice. However, DTs completing private appointments were also more able to use direct access to create their own treatment plan and therefore work to their full scope of practice. 
Changing patient demands prompt a reconsideration of the current dentistry model, providing a needs-based system, improving patient outcomes and with appropriate regulations and financial incentives. ${ }^{26}$ While this paper has focussed on the contributions of the role of one dental team member in general practice, we are not proposing that their contribution is "better" than any others, we have merely explored one approach to skill-mix, one prompted by recent regulation changes. Turner, Tripathee and MacGillivray ${ }^{30}$ explain that new models need to explore the question "how can we do our best?" rather than "who does what best?" to ensure clinical quality.

\section{Conclusion}

Practices with DTs provided a more preventive-focussed approach to patient-care and enabled the dentists in these practices to perform more complex work than in those without. Positive patient satisfaction and confidence in practitioners' ability across practices suggests patient acceptability of a preventive model delivered by well-trained members of the dental team. While both types of practices were operating well in terms of patient satisfaction, the results support suggestions for reconfiguration of the dental workforce away from expensive, pressured, 'top-heavy’ staffing, towards a redistributed workforce allowing both dentists and DCPs to carry out their full scope of practice. However, funding mechanisms must be in place to support access for NHS patients - arguably the population who would benefit most.

\section{Declaration of interests}

The project was part of a wider study funded by Health and Care Research Wales. 


\section{References}

1. Macey, R, Glenny, AM, Brocklehurst, P. (2016) Feasibility study: assessing the efficacy and social acceptability of using dental hygienist-therapists as front-line clinicians. British Dental Journal.221: 11:717-21.

2. Harper, P, Kleinman, E, Gallagher, J, Knight, V. (2013) Cost-effective workforce planning: Optimising the dental team skill-mix for England. Journal of Enterprise Information Management.26: 1:91-108.

3. Bullock, A, Firmstone, V. (2011) A professional challenge: the development of skill-mix in UK primary care dentistry. Health services management research : an official journal of the Association of University Programs in Health Administration / HSMC, AUPHA.24: 4:190-5.

4. Gallagher, JE, Wilson, NHF. (2009) The future dental workforce? British Dental Journal.206: 4:195-99.

5. General Dental Council. Scope of practice. September 2013. London: General Dental Council, 2013.

6. Evans, C, Chestnutt, IG, Chadwick, BL. (2007) The potential for delegation of clinical care in general dental practice. British Dental Journal.203: 12:695-99.

7. Macey, R, Glenny, A, Walsh, T, Tickle, M, Worthington, H, Ashley, J, et al., (2015) The Efficacy of Screening for Common Dental Diseases by Hygiene-Therapists: A Diagnostic Test Accuracy Study. Journal of Dental Research.94: 3:70S-78S.

8. Wanyonyi, KL, Radford, DR, Harper, PR, Gallagher, JE. (2015) Alternative scenarios: harnessing mid-level providers and evidence-based practice in primary dental care in England through operational research. Human Resources for Health.13.

9. General Dental Council. (2013) Guidance on Direct Access.

10. Turner, S, Ross, M. (2017) Direct access: how is it working? British Dental Journal.222: 3:191-

97.

11. National Institute for Health and Care Excellence. Guideline MPG2: Patient Group Directions 2013 [cited 2018]. Available from: https://www.nice.org.uk/guidance/mpg2.

12. Wilson, M, Lewney, J. Piloting Direct Access in the Community Dental Services in Wales; a review of guidelines and practical considerations. DENTALHEALTH:36.

13. Sandom, F. (2017) Is regulation hampering direct access? Bdj Team.4:17031.

14. Jones, G, Evans, C, Hunter, L. (2008) A survey of the workload of dental therapists/hygienisttherapists employed in primary care settings. British Dental Journal.204: 3.

15. Ross, MK, Ibbetson, RJ, Turner, S. (2007) The acceptability of dually-qualified dental hygienist-therapists to general dental practitioners in South-East Scotland. British Dental Journal.202: 3.

16. Ward, P. (2006) The changing skill mix - experiences on the introduction of the dental therapist into general dental practice. British Dental Journal.200: 4:193-97.

17. Department of Health. Dental contract reform: Engagement. London: Department of Health, , 2014.

18. Department of Health. Dental contract reform. Overview document. 2015.

19. Harris, R, Burnside, G. (2004) The role of dental therapists working in four personal dental service pilots: type of patients seen, work undertaken and cost-effectiveness within the context of the dental practice. British Dental Journal.197: 8:491-96.

20. NHS Wales/Wales Government. Making Prudent Healthcare Happen 2014. Available from: http://www.prudenthealthcare.org.uk/.

21. National Maternal and Child Oral Health Resource Center. Safety Net Dental Clinic Manual Patient Satisfaction Survey 2011 [28.07.17]. Available from:

http://dentalclinicmanual.com/docs/Patient Survey3.pdf.

22. Betsi Cadwaladr University Health Board. All Wales Patient Experience Framework 2013 [28.07.17]. Available from: http://www.wales.nhs.uk/sitesplus/861/opendoc/217526. 
23. Bonehill, J. (2013) Direct access to dental treatment: understanding the pros and cons. Dental Nursing.9: 9:528-31 4p.

24. Williams, DM, Medina, J, Wright, D, Jones, K, Gallagher, JE. (2010) A review of effective methods of delivery of care: skill-mix and service transfer to primary care settings. Primary dental care : journal of the Faculty of General Dental Practitioners (UK).17: 2:53-60.

25. Richards, D. (2011) Skill-mix and service transfer to primary care settings. Evidence-based dentistry.12: 2:51-51.

26. Brocklehurst, P, Macey, R. (2015) Skill-mix in preventive dental practice - will it help address need in the future? BMC oral health.15 Suppl 1:S10-S10.

27. Mathur, MR, Singh, A, Watt, R. (2015) Addressing inequalities in oral health in India: need for skill mix in the dental workforce. Journal of family medicine and primary care.4: 2:200-2.

28. Yang, T, Chen, B, Wanchek, T. (2017) Dental Therapists: A Solution to a Shortage of Dentists in Underserved Communities? J Public Health Reports.285-288: 132:3.

29. Gallagher, JL, Wright, DA. (2003) General dental practitioners' knowledge of and attitudes towards the employment of dental therapists in general practice. British Dental Journal.194: 1:37-41. 30. Turner, S, Tripathee, S, MacGillivray, S. (2013) Direct access to DCPs: what are the potential risks and benefits? British Dental Journal.215: 11:577-82.

31. Sun, N, Burnside, G, Harris, R. (2010) Patient satisfaction with care by dental therapists. British Dental Journal.208: 5.

32. Ross, M, Ibbetson, R, Turner, S. (2007) The acceptability of dually-qualified dental hygienisttherapists to general dental practitioners in South-East Scotland. British Dental Journal.202: 3:online E8.

33. Jones, G, Devalia, R, Hunter, L. (2007) Attitudes of general dental practitioners in Wales towards employing dental hygienist-therapists. British Dental Journal.203: 9.

34. Harris, RV, Sun, N. (2012) Translation of remuneration arrangements into incentives to delegate to English dental therapists. Health Policy.104: 3:253-59.

35. Brocklehurst, PR, Tickle, M. (2011) Is skill mix profitable in the current NHS dental contract in England? British Dental Journal.210: 7:303-08.

36. Sun, N, Harris, RV. (2011) Models of practice organisation using dental therapists: English case studies. British Dental Journal.211: 3.

37. Williams, SA, Bradley, S, Godson, JH, Csikar, JI, Rowbotham, JS. (2009) Dental therapy in the United Kingdom: Part 3. Financial aspects of current working practices. British Dental Journal.207: 10:477-83.

38. Wales Government. Taking Oral Health Improvement and Dental Services Forward in Wales A Framework outlining priorities for dentistry and a future work programme 2017 [04.08.2017].

Available from: http://gov.wales/docs/phhs/publications/170328oralhealthen.pdf. 